Received: 22 November 2018

Accepted: 24 August 2019

Published online: 03 October 2019

\section{Micro-scale modelling of the urban wind speed for air pollution applications}

\author{
Thor-Bjørn Ottosen $\mathbb{1}^{1,2,3}{ }^{2}$, Matthias Ketzel $\mathbb{1}^{4,5}$, Henrik Skov ${ }^{2,4}$, Ole Hertel ${ }^{4}$, Jørgen Brandt ${ }^{4}$ \& \\ Konstantinos E. Kakosimos $\mathbb{D}^{3}$
}

Modelling wind speeds in urban areas have many applications e.g. in relation to assessment of wind energy, modelling air pollution, and building design and engineering. Models for extrapolating the urban wind speed exist, but little attention has been paid to the influence of the upwind terrain and the foundations for the extrapolation schemes. To analyse the influence of the upwind terrain and the foundations for the extrapolation of the urban wind speed, measurements from six urban and nonurban stations were explored, and a model for the urban wind speed with and without upwind influence was developed and validated. The agreement between the wind directions at the stations is found to be good, and the influence of atmospheric stability, horizontal temperature gradients, land-sea breeze, temperature, global radiation and Monin-Obukhov Length is found to be small, although future work should explore if this is valid for other urban areas. Moreover, the model is found to perform reasonably well, but the upwind influence is overestimated. Areas of model improvement are thus identified. The upwind terrain thus influences the modelling of the urban wind speed to a large extent, and the fundamental assumptions for the extrapolation scheme are fulfilled for this specific case.

The urban wind speed has been an area of research, as part of urban climatology studies, since the $1930 \mathrm{~s}^{1}$. The initial research interest focused on large-scale effects ${ }^{2,3}$ and was subsequently spurred by the interest in the problems of air pollution ${ }^{4,5}$. The topic of urban wind speed for air pollution applications has remained an active area of research until today ${ }^{6-11}$. In recent years, the urban wind speed has as well been of interest for urban wind energy (e.g. ${ }^{12-14}$ ) or assessments of wind loadings on tall buildings ${ }^{15}$.

Often, financial and technical constraints prevent the use of measurements to assess the urban wind speed. Models of the urban wind speed are therefore often used to estimate the urban wind speed at many locations. The present study is motivated by the use of the modelled urban wind speed as input to regulatory air pollution models, since previous work ${ }^{16,17}$ have shown the importance of this input. These models are characterised by being fast, generally applicable, reliable, and simple to use ${ }^{18-20}$. The same principles continue to guide the development of such models even today despite the evolution of high performance computing facilities. This is the case, since the needs have evolved and increased as well, demanding longer and finer time scales and more and diverse receptor locations. In line with this approach, focus of the present study is on modelling hourly averaged wind speeds in the roughness sublayer (using the terminology of ${ }^{21}$ ), since this is the averaging time and relevant height used in regulatory air quality models.

The urban wind speed can be modelled using different approaches:

1. Large-scale numerical weather prediction models (e.g. $\left.{ }^{22,23}\right)$.

2. Physical models (e.g. wind or water tunnels) (e.g. $\left.{ }^{24}\right)$.

3. Computational fluid dynamics (CFD) models (e.g. ${ }^{25,26}$ ).

4. Models based on analytical horizontal and vertical extrapolations (e.g. the references in Table 4 in the Supplementary Material).

${ }^{1}$ Institute of Science and the Environment, University of Worcester, Worcester, UK. ${ }^{2}$ Department of Chemical Engineering, Biotechnology and Environmental Technology, University of Southern Denmark, Odense, Denmark. ${ }^{3}$ Department of Chemical Engineering, Texas A\&M University at Qatar, Doha, Qatar. ${ }^{4}$ Department of Environmental Science, Aarhus University, Roskilde, Denmark. ${ }^{5}$ Global Centre for Clean Air Research (GCARE), University of Surrey, Guildford, GU2 7XH, United Kingdom. Correspondence and requests for materials should be addressed to K.E.K. (email:k.kakosimos@qatar.tamu.edu) 


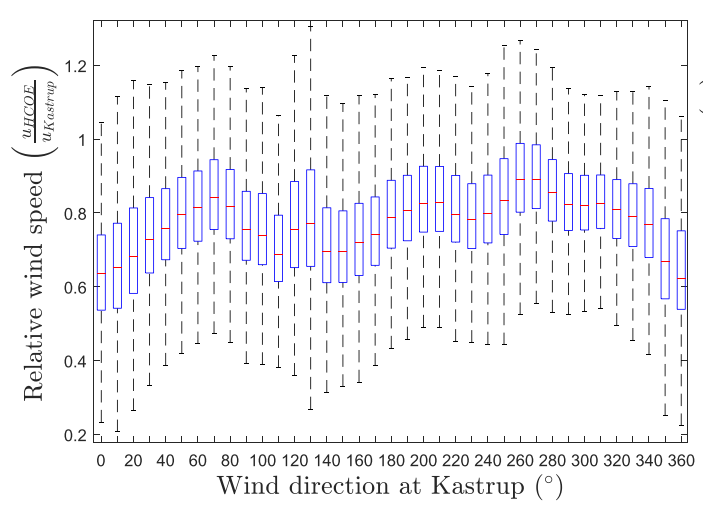

(a)

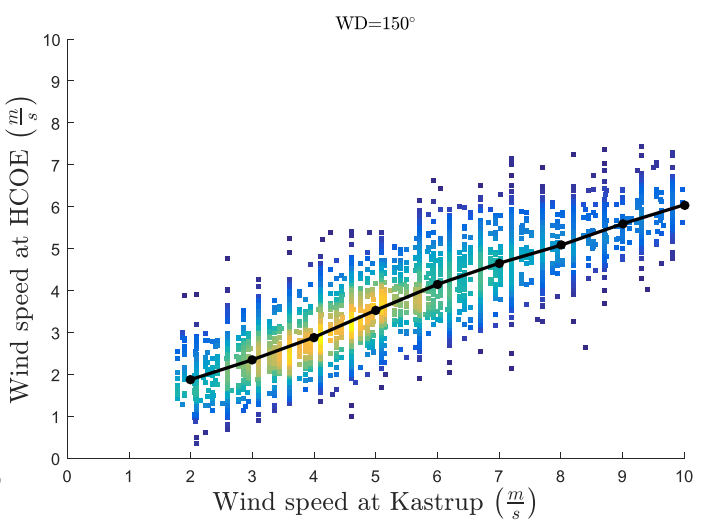

(b)

Figure 1. Boxplot of the relative wind speed between HCOE (urban mast) and Kastrup (airport) (1a) and scatterplot of the wind speed at HCOE versus the wind speed at Kastrup for a specific wind direction in Kastrup of $150^{\circ}(\mathbf{l b})$. The red lines in (a) are the medians, the boxes are the 25 and 75 percentiles, and the whiskers are $\approx 2.7 \sigma$. The colors in (b) represent the relative density of points. Wind speeds below $1.75 \mathrm{~m} \mathrm{~s}^{-1}$ and above $10 \mathrm{~m} \mathrm{~s}^{-1}$ in Kastrup have been removed.

The design principles behind regulatory air quality models effectively limits the models in the present context to models applying analytical horizontal and vertical extrapolations.

A number of recent models of the urban wind speed using analytical horizontal and vertical extrapolations have been presented in the literature $e^{7,12,13,15,27-30}$. An overview of recent models of the urban wind speed using analytical horizontal and vertical extrapolations are given in Table 4 in the Supplementary Material. All studies are using either input from a nearby airport or various wind speed databases as input. The extrapolation method termed Profile in Table 4 is using a double vertical-horizontal interpolation following the approach of ${ }^{31}$. The studies in Table 4 are using logarithmic profiles, whose application and validity are discussed in more detail in the Supplementary Material. The extrapolation method termed $I B L$ (Internal Boundary Layer) is using an extrapolation approach across multiple internal boundary layers following either ${ }^{32}$ and $^{33}$ or $^{34}$. Apart from the studies in Table $4^{35}$, fitted straight lines to scatter plots of measured rural and urban wind speed data from Birmingham, UK $(2 \times 4$ weeks); Copenhagen, Denmark (1 year); Lisbon, Portugal ( 3 months); and Barcelona, Spain (1 year). Their study was part of European Cooperation in Science and Technology (COST) $715^{6}$ and concluded that the relationship was site specific and required more investigation. The many different approaches presented in Table 4 highlights the need for model comparisons; however, only a few model comparisons are available in the literature $^{36}$, and given the vast number of approaches a comparison is beyond the scope of the present study.

Previous work on the influence of the upstream terrain on urban wind speed measurements was reviewed, and a variation of a factor of two in the drag coefficient for approximately $20^{\circ}$ wind direction change was shown ${ }^{37}$. This development in measurements has, to the best of the authors' knowledge, not been reflected in the modelling. The influence of the upwind terrain above the blending height has been modelled ${ }^{13}$, and the upwind influence as a function of four wind directions (North, South, East, and West) has been modelled as well ${ }^{28}$. As seen from Table 4 in the Supplementary Material, neither of these studies have performed validation on the hourly time scale, the time scale of relevance for the present study, and the accuracy of these modelling approaches, for this time scale, is thus unknown. Moreover, the studies in Table 4 in the Supplementary Material have furthermore paid less attention to whether the fundamental assumptions for the extrapolation schemes are fulfilled. It is thus an open question, to what extent the upwind terrain influences modelling of the urban roughness sublayer wind speed, and to what extent, the assumptions for the extrapolation technique are fulfilled.

To asses the validity of the assumptions for the extrapolation technique, exploratory data analyses of meteorological data were performed. To assess the influence of the upwind terrain in models of the urban wind speed, a new model for the urban wind speed, with (henceforth referred to as the advanced model) and without (henceforth referred to as the simple model) upwind influence, was developed and validated using the measurements. Given the challenge of this task, with respect to both modelling and measurements, the present model should be considered a first step in the direction of a more generally applicable urban wind speed model.

\section{Results}

Analysis of fundamental model assumptions. Influence of wind speed and direction. The wind speed extrapolation model uses only the wind direction and wind speed as input data. That the relative wind speed is wind direction dependent is evident from the box plot in Fig. 1. Whereas the median value is changing with wind direction, the scatter in the relative wind speed appears to be independent of wind direction. As an example, the wind speed at $\mathrm{HCOE}$ is plotted against the wind speed in Kastrup for an arbitrary wind direction of $150^{\circ}$ in Fig. 1. It is evident from the figure that the relative wind speed is wind speed dependent. It can thus be confirmed from Fig. 1 that the extrapolated wind is dependent on wind speed and wind direction.

It is moreover evident from Fig. 1 that the wind speed at the HCOE (urban background station at the H. C. Oersteds Institute, University of Copenhagen. See Supplementary Material for station characteristics) is not constant for a given input wind speed and a given wind direction. Instead, for each input wind speed and direction, 


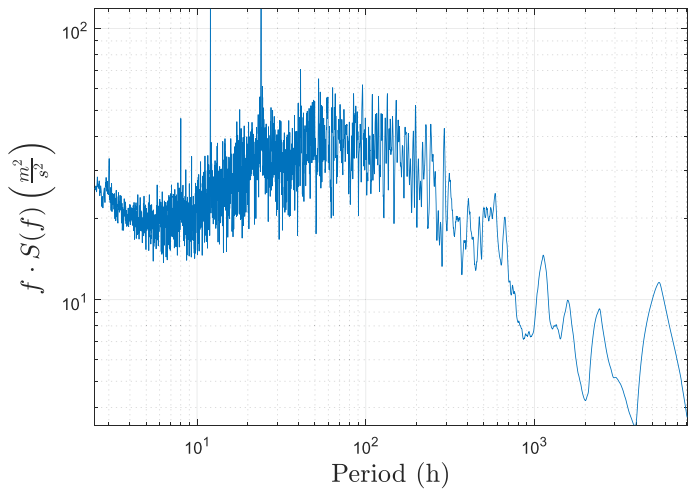

(a)

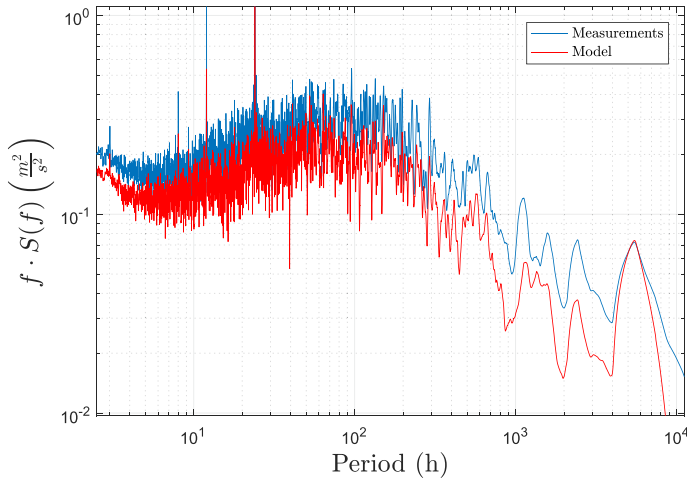

(b)

Figure 2. Lomb-Scargle periodogram for the covariance of the wind speed between Kasptup and HCOE (1a)

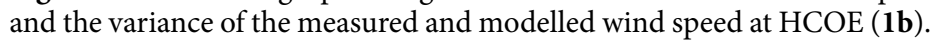

there is a distribution of wind speeds at HCOE. This distribution in wind speeds is caused by turbulence in the atmosphere at all length scales between the two stations. Since this variance is not modelled in the present application, the model validation will be divided into two parts: To assess the performance of the model, it is validated against the median values for each wind speed and wind direction. The median is chosen instead of the mean since the distributions are not always symmetric. To explore the predictability of the hourly wind speed, the model is compared to the full dataset.

Influence of horizontal temperature gradient and other effects. Horizontal temperature gradients will cause poor performance of this scheme, since this will cause a change in pressure gradient with height ${ }^{31}$. The influence of horizontal temperature gradient was examined by analysing the relative wind speed for the Copenhagen and Aarhus airport-urban mast station pairs as a function of temperature difference between the two stations. The analysis showed that the mean temperature in Aarhus is $0.88^{\circ} \mathrm{C}$ higher than the airport with a standard deviation of $1.53^{\circ} \mathrm{C}$. For Copenhagen the mean and standard deviation is respectively $0.33^{\circ} \mathrm{C}$ and $1.15^{\circ} \mathrm{C}$. These are small temperature differences between the stations which also explains why the analysis showed almost no effect of horizontal temperature gradient.

The presence of land-sea breezes was examined by plotting the diurnal variation in the wind direction at Kastrup Airport. The station was found not to be influenced by land-sea breeze.

In an attempt to explain the remaining variance in the measurements, scatter plots of the remaining variance were produced and these showed only vague connections to the other meteorological variables measured (being temperature and global radiation $)^{38}$. No connection was likewise found between the remaining variance and the atmospheric stability represented by the Monin-Obukhov Length (the height at which the production of turbulence by mechanical and buoyancy forces is equal ${ }^{39}$, [p. 747]) calculated based on meteorological data from Meteorological Model 5 (air density, temperature, friction velocity and surface heat flux) on a $5.6 \mathrm{~km} \times 5.6 \mathrm{~km}$ resolution for the year 2012. This indicates that wind speed and wind direction are the determining variables among the inputs available.

The analysis of the agreement in wind direction between the stations and the influence of atmospheric stability can be found in the Supplementary Material.

The above analyses show that the present case study is dominated by mesoscale weather patterns, which allows the use of the presented extrapolation scheme. Other cities have reported temperature gradients up to $8^{\circ} \mathrm{C}^{40}$, and mixed results have been reported as to the influence of atmospheric stability in urban areas ${ }^{37,41-43}$. In these cases, a more advanced modelling approach might be required.

Analysis of the spectral representation of data. To analyse the properties of the different ways to represent wind speed data for two stations (absolute wind speeds, relative wind speeds, or wind speed differences), the spectral representation was analysed. In this section, the term model is used to mean the advanced model, since the simple model will have the same spectra as the measurements. Since HCOE is the longest time series in the present study, the periodogram of the covariance between the wind speed at this station and the wind speed in Kastrup is shown in Fig. 2. It is evident that the two stations have high covariance at the frequencies corresponding to the diurnal variation plus the harmonics of this frequency. Moreover, there is an annual cycle seen in the wind speed. Naturally, these wind speed cycles will influence the stations in the same way owing to the nearness of the stations to each other. It can also be seen that the covariance periodogram is dominated by frequencies in the range $\approx 20 \mathrm{~h}$ to $\approx 130 \mathrm{~h}$. This is the time scale for changes in the weather pattern on the mesoscale. This is also seen in Fig. 2 where it is evident that both the model and the measurements have the same spectrum.

The periodogram of the relative wind speed is shown in Fig. 3. The peaks for the diurnal and annual variation can also be found in this spectrum. The $y$-axes in Fig. 2 are different since the total variance of the relative wind speed is much smaller than the total covariance of the two wind speeds, as shown in Fig. 2. However, it is shown from this figure that the mesoscale effects have been diminished, as seen from the absence of high values in the $10 \mathrm{~h}$ to $100 \mathrm{~h}$ range. The local scale phenomena are now dominating the variance in the representation, which is 


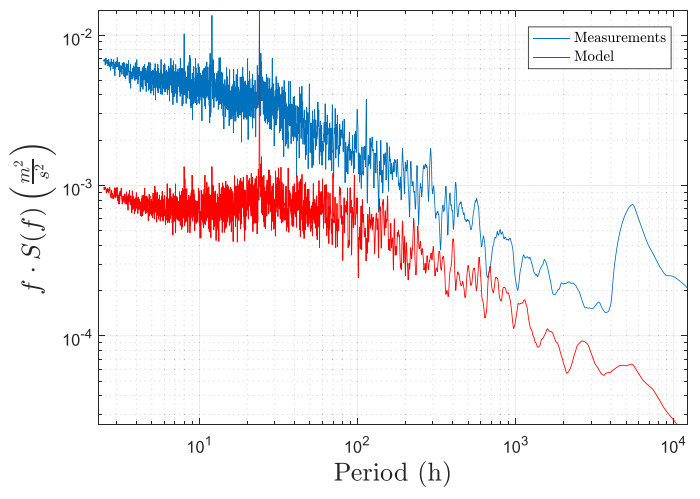

(a) Relative wind speeds

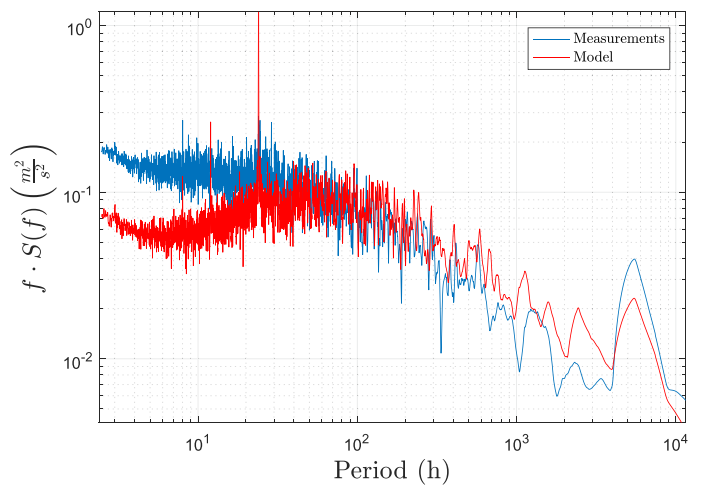

(b) Wind speed differences

Figure 3. Lomb-Scargle periodogram of the variance of the relative wind speed (3a) and the wind speed differences (3b) between Kastrup and HCOE for both the model and the measurements.

desirable as this is the focus of the present model. Unfortunately, this pattern is not reproduced in the model output, which invalidates this representation for model validation. The same phenomenon is seen for the wind speed difference in Fig. 3. The explanation is that the absolute wind speeds are used as input to the model. Since these depend heavily on mesoscale effects, the mesoscale effects will also be present in the output. The representation where the model output and the measurements have the same spectrum is therefore the absolute wind speeds. This representation is therefore used in the model validation.

Figure 2 showed that the wind speed data is not identically distributed due to the periodicities in data, and since data are strongly autocorrelated, the wind speed data is not independent. To the best of the authors knowledge, there is no standard way to transform wind speed data into an independent and identically distributed dataset. Since independent and identically distributed data is a fundamental assumption for several statistical analyses (e.g. correlation, analysis of variance (ANOVA), etc.) this effectively limits the ways the data can be analysed.

Model validation. Validation against median values. The model output and the measurements as a function of wind direction can be seen in Fig. 4. As seen from Fig. 4, both models predict the large wind speed reduction from the airport to the city and the smaller wind speed reduction from the mast to the roof. The curves for the median input wind speed are not identical since each one cover different periods in time for respectively the permanent stations and the campaign stations. The figures also show that both models tend to underestimate the wind speed, more so for the advanced model than the simple model.

For Kastrup $\rightarrow$ HCOE the advanced model reproduces the wind direction dependency with good performance. HCOE is, as seen in the maps in the attached Google maps file, influenced by a large park for easterly winds and subsequently influenced by a large high rise building at $140^{\circ}$ to $150^{\circ}$; these features are nicely reproduced by the advanced model and not seen in the simple model. This pattern is, to some extent, also seen for $\mathrm{HCOE} \rightarrow$ Jagtvej (urban roof level station at Jagtvej, Copenhagen. See Supplementary Material for station characteristics) and $\mathrm{HCOE} \rightarrow \mathrm{HCAB}$ (urban roof level station at H. C. Andersens Boulevard, Copenhagen. See Supplementary Material for station characteristics) and in Kastrup $\rightarrow$ Jagtvej due to the short distance between HCOE and Jagtvej. Both the advanced model and the simple model are underestimating the wind speed for westerly wind directions, more so for the simple model than the advanced model for this station. This can potentially be connected to some areas of detached houses upstream of the Kastrup station whose influence is underestimated in both models. This is emphasised by the fact that all models underestimate westerly wind speeds when Kastrup is used as input.

$\mathrm{HCAB}$ is influenced by an amusement park with correspondingly low roughness for south-westerly wind directions thus giving a characteristic wind direction pattern. This pattern is overestimated in the advanced model for $\mathrm{HCOE} \rightarrow \mathrm{HCAB}$ and not present in the simple model. The underestimation for Kastrup $\rightarrow$ Jagtvej for south-westerly winds can potentially be linked to something (probably the aforementioned detached houses upwind of the Kastrup station) not accurately modelled in Kastrup. This is the case since the wind direction pattern is nicely reproduced by the model when $\mathrm{HCOE}$ is used as input. $\mathrm{HCOE} \rightarrow$ Jagtvej is nicely reproduced by both models. This means that this represents a particularly simple situation where a simpler approach can be useful. The reason is the short distance between the stations meaning that the wind speed and direction will be extremely identical for the two stations.

Tirstrup $\rightarrow$ Aarhus (urban background station at the Aarhus town hall, Aarhus. See Supplementary Material for station characteristics) is a special case since the airport wind speed is only slightly larger than the urban wind speed. For a small interval around $200^{\circ}$ the urban wind speed is actually larger than the airport wind speed. This is the case since the urban station is only a few meters higher than the airport station. Moreover, the airport station is influenced by forest areas close to the station. Nevertheless, the model underestimation for the advanced model is more pronounced for this station compared with the other stations. For the interval $\left[0^{\circ}: 150^{\circ}\right]$ the simple model is reproducing observations fairly well whereas the forest areas for southern wind directions are underestimated in both models, more so for the advanced model than the simple model. 


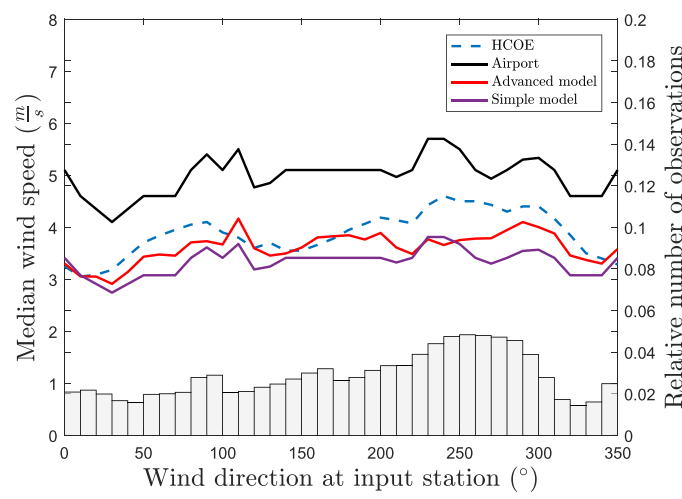

(a) Kastrup $\rightarrow \mathrm{HCOE}$ (17 years)

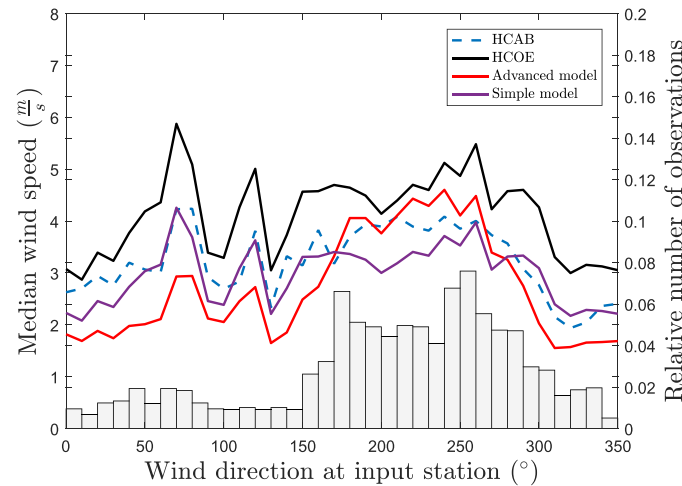

(c) $\mathrm{HCOE} \rightarrow \mathrm{HCAB}$ (5 months)

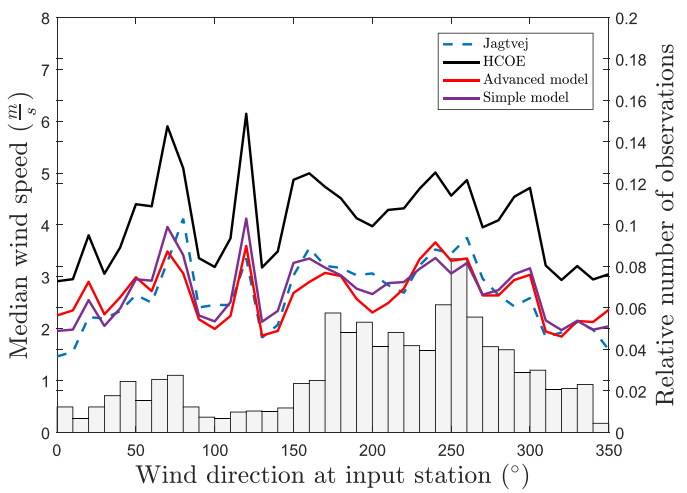

(e) HCOE $\rightarrow$ Jagtvej (5 months)

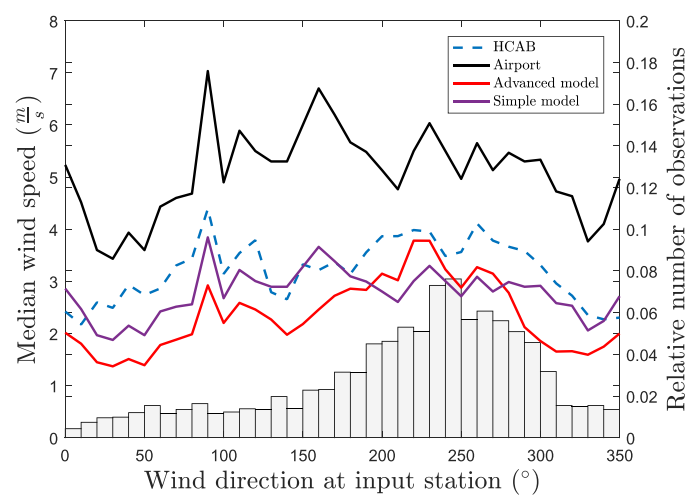

(b) Kastrup $\rightarrow \mathrm{HCAB}$ (5 months)

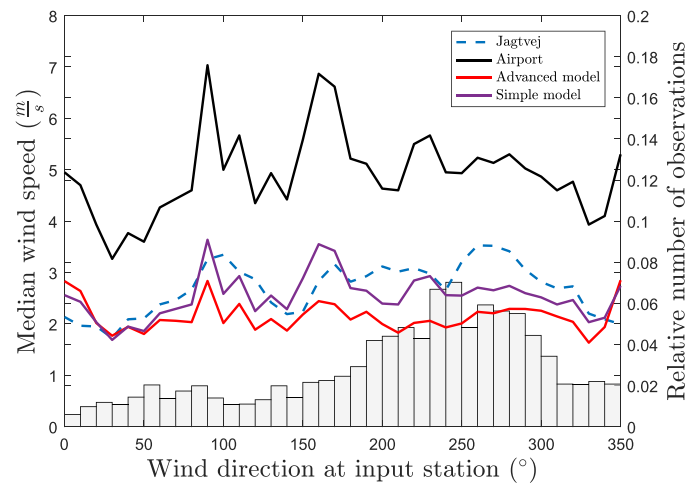

(d) Kastrup $\rightarrow$ Jagtvej (5 months)

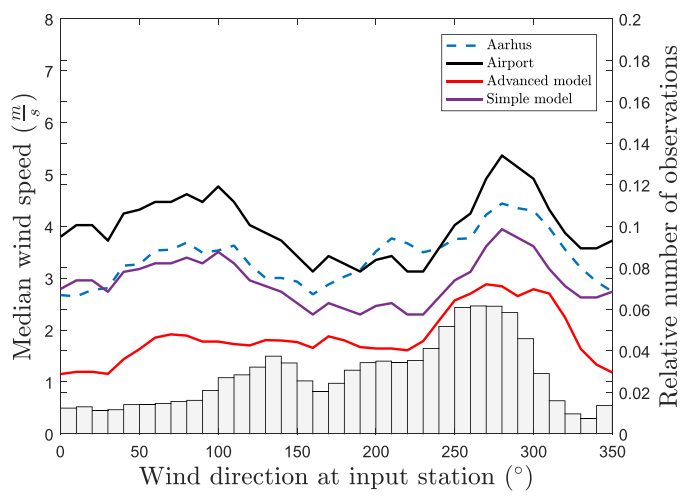

(f) Tirstrup $\rightarrow$ Aarhus (10 years)

Figure 4. Plot of the median wind speed for the measurements (blue, dashed), the advanced model (red), the simple model (purple,) and the input wind speed (black) (either the closest mast or airport station) as a function of wind direction for the three stations in Copenhagen using the two available input stations plus Aarhus using Tirstrup Airport as input. The bar charts represent the relative number of observations for each wind direction and are plotted on the right y-axis.

The comparison between the simple model and the advanced model indicates that the upwind influence is overestimated in the advanced model for some wind direction intervals. This can be seen in e.g. Kastrup $\rightarrow \mathrm{HCAB}$ $\left[300^{\circ}: 350^{\circ}\right]$ and $\mathrm{HCOE} \rightarrow \operatorname{HCAB}\left[50^{\circ}: 150^{\circ}\right]$. It is characteristic that these wind direction intervals are having few observations as seen in the bar charts in Fig. 4. The effect of this is that the curve is disproportionately influenced by individual weather events creating e.g. the spikes in the curves for both Jagtvej and HCAB. It is likely that the wind direction plot for the urban mast stations will change as the time series expand and the spikes become smoothed out. It is therefore too early to conclude on the upwind influence in the model for these stations. The effect is also pronounced for Aarhus where the simple model is almost perfect for $\left[0^{\circ}: 150^{\circ}\right]$. Future work should aim at reducing the upwind influence for this station. On the other hand, it is shown that the simple approach is insufficient for stations with inhomogeneous upwind roughness such as Kastrup, HCOE, and HCAB.

A similar pattern can be seen in Table 1. In general both models have smaller relative deviations (except the advanced model for Aarhus) compared with the input. The simple model has in general a smaller relative deviation indicating the previously described problems with the upwind influence in the model. On the other hand, 


\begin{tabular}{|l|l|l|l|l|l|}
\hline Receptor & Model & $\begin{array}{l}\text { Max } \\
(\%)\end{array}$ & $\begin{array}{l}\text { Min } \\
(\%)\end{array}$ & $\begin{array}{l}\text { Mean } \\
(\%)\end{array}$ & $\begin{array}{l}\text { Correlation } \\
\text { coefficient }\left(\boldsymbol{R}^{2}\right)\end{array}$ \\
\hline HCOE & Kastrup & 57 & 11 & 30 & 0.67 \\
\hline HCOE & Advanced model & 20 & 0 & 8 & 0.74 \\
\hline HCOE & Simple model & 26 & 1 & 14 & \\
\hline HCAB & Kastrup & 116 & 23 & 61 & 0.63 \\
\hline HCAB & Advanced model & 49 & 4 & 27 & 0.80 \\
\hline HCAB & Simple model & 33 & 0 & 17 & \\
\hline Jagtvej & Kastrup & 165 & 46 & 86 & 0.58 \\
\hline Jagtvej & Advanced model & 43 & 2 & 24 & 0.12 \\
\hline Jagtvej & Simple model & 37 & 1 & 13 & \\
\hline HCAB & HCOE & 55 & 6 & 29 & 0.85 \\
\hline HCAB & Advanced model & 44 & 1 & 22 & 0.84 \\
\hline HCAB & Simple model & 23 & 1 & 11 & \\
\hline Jagtvej & HCOE & 99 & 24 & 57 & 0.85 \\
\hline Jagtvej & Advanced model & 54 & 0 & 14 & 0.76 \\
\hline Jagtvej & Simple model & 33 & 0 & 12 & \\
\hline Aarhus & Tirstrup & 52 & 0 & 21 & 0.64 \\
\hline Aarhus & Advanced model & 59 & 28 & 46 & 0.84 \\
\hline Aarhus & Simple model & 37 & 0 & 14 & \\
\hline
\end{tabular}

Table 1. Maximum, minimum, and mean absolute relative deviation among the median values as a function of wind direction of the input, and the two models. The simple model has the same correlation as the input and is therefore not shown.

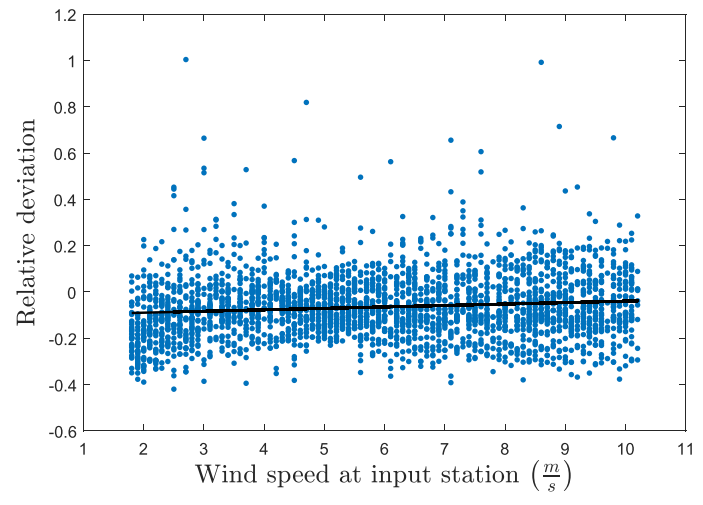

(a) Advanced model

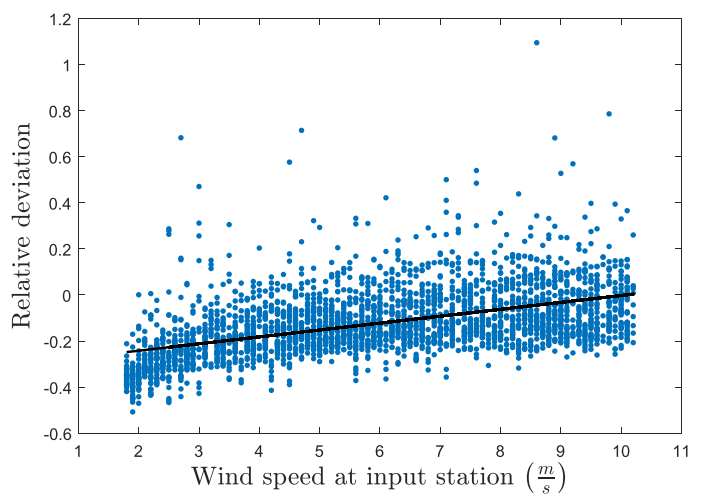

(b) Simple model

Figure 5. Plot of the relative deviation between the model and the measurements as a function of input wind speed. The black line is a linear trend line.

the advanced model generally have a higher correlation coefficient indicating a stronger association between the results from the advanced model and the measurements. It can be seen that the correlation is dropping for Jagtvej. It has to be noted that the correlation between HCOE and Jagtvej is already quite high, and it is thus a challenge to improve on this figure. This shows a need for a more advanced model for longer extrapolation distances. For Kastrup $\rightarrow$ Jagtvej the low correlation is explained by the urban station being, as seen from the attached Google Maps file, located in quasi-homogeneous surroundings also leading to a lower variance for this station. This means that the correlation coefficient is a skewed performance measure for this combination of stations.

To analyse the dependency on wind speed, the relative deviation $\left(\frac{u_{\text {model }}-u_{\text {measurements }}}{u_{\text {measurements }}}\right)$ was calculated for each wind speed and wind direction. A linear trend was subsequently fitted to these data. A perfect model will have a slope and intercept of zero. An example of this for Kastrup $\rightarrow$ HCOE is shown in Fig. 5. The slope and intercept for the trend line for all the streets for both models can be found in Table 2. From Table 1 the advanced model appeared to be somewhat better than the simple model for Kastrup $\rightarrow$ HCOE. From Fig. 5 it is evident that the median performance of the simple model covers some significant differences between high and low wind speeds, with the bias on the low wind speeds being up to $30 \%$. This is not seen for the advanced model, where the bias is fairly homogeneously distributed with respect to wind speed.

A similar trend can be seen for the slope and intercept of the trend line for the extrapolations in Table 2. This indicates that using the modelled urban boundary layer height for horizontal extrapolation performs better than assuming a constant extrapolation height of $4 h_{r}$. For HCOE $\rightarrow$ Jagtvej the performance of the two models is 


\begin{tabular}{|l|l|l|l|l|}
\hline Receptor & Model & Input & Slope $\left(\mathbf{1 0}^{-\mathbf{2}}\right)$ & Intercept \\
\hline HCOE & Advanced model & Kastrup & 0.62 & -0.10 \\
\hline HCOE & Simple model & Kastrup & 2.99 & -0.30 \\
\hline HCAB & Advanced model & Kastrup & 1.97 & -0.34 \\
\hline HCAB & Simple model & Kastrup & 3.94 & -0.33 \\
\hline Jagtvej & Advanced model & Kastrup & 0.38 & -0.20 \\
\hline Jagtvej & Simple model & Kastrup & 2.95 & -0.17 \\
\hline HCAB & Advanced model & HCOE & 2.85 & -0.24 \\
\hline HCAB & Simple model & HCOE & 0.94 & -0.10 \\
\hline Jagtvej & Advanced model & HCOE & -0.15 & 0.01 \\
\hline Jagtvej & Simple model & HCOE & 0.16 & 0.02 \\
\hline Aarhus & Advanced model & Tirstrup & 1.21 & -0.52 \\
\hline Aarhus & Simple model & Tirstrup & 4.75 & -0.33 \\
\hline
\end{tabular}

Table 2. Slope and intercept of a linear function fitted to the relative deviation between model and measurements as a function of input wind speed.

approximately equal whereas for $\mathrm{HCOE} \rightarrow \mathrm{HCAB}$ the simple model performs better. This can be explained by the large low-roughness areas (large parks/amusement parks) close to these two stations influencing $u_{*}$. Future work should aim at reducing this effect in the advanced model.

Validation against the full dataset. The histograms of the residuals between the input and the measurements and the results from the models and the measurements can be seen in Fig. 6 . In general, the models are having smaller and more narrow bias compared with the input. Figure 6 shows mixed results with the advanced model being a little bit better for Kastrup $\rightarrow \mathrm{HCOE}$ and $\mathrm{HCOE} \rightarrow \mathrm{HCAB}$, only small differences between the two models for Kastrup $\rightarrow \mathrm{HCAB}$ and $\mathrm{HCOE} \rightarrow$ Jagtvej, whereas the simple model performs better for Kastrup $\rightarrow$ Jagtvej and Tirstrup $\rightarrow$ Aarhus. In general, this is in line with the results from the validation against median values where the reasons have also been discussed.

As can be seen, the longer the extrapolation distance the wider the distributions. This is again caused by turbulence in the atmosphere on all length scales between the two stations. Reducing the spread further will be challenging given that this turbulence is not measured in the present project.

\section{Conclusion}

The upwind terrain influences the modelling of the urban wind speed to a large extent. Modelling this effect is a significant challenge which in the present paper has been met to some extent. The model results show (except Aarhus) good agreement between the models and the measurements compared to model results given in e.g. ${ }^{16}$ for an atmospheric dispersion model.

The influence of the upwind terrain was shown to be overestimated in the model, and future work should aim at reducing this effect. Considering that the model contains many uncertain parameters, this result is acceptable.

The agreement in wind direction between the stations were shown to be high. Moreover, the influence of atmospheric stability, horizontal temperature gradients, land-sea breeze, temperature, global radiation, and Monin-Obukhov Length were shown to be small. One remaining question is whether this finding is only valid for the present location or it represents a more general phenomenon.

Many alternative parametrizations to the ones selected in the present study exist. To direct future research to the parametrizations with the largest influence on the model, sensitivity analyses, in line with the ones presented in $^{16}$, could be performed. This could subsequently form the basis for studies on the comparison of the available parametrizations. Likewise, comparison studies between different types of models (e.g. numerical weather presiction models, multivariate linear regression models etc.) would be relevant to highligt strengths and weaknesses of different modelling approaches.

In the present study, the urban areas are assumed to be flat although the height above sea level varies by several tens of meters. The influence of the urban topography is likewise a potential area for a future study.

Modelling the wind speed in the urban canopy layer is complicated and several parametrizations has been proposed in the literature. One remaining question is the validity of these parametrizations, where measurements in the canopy layer, wind tunnel studies, and the use of CFD (Computational Fluid Dynamics) models could contribute to answer this question.

\section{Methods}

In the analyses of the present study, wind speeds below $2 \mathrm{~m} \mathrm{~s}^{-1}$ have been removed (with the exception of the classification into atmospheric stability classes). This was done to avoid the difficulties of the anemometer start up speeds, and since these data will be disproportionately influenced by turbulence. Moreover, the given uncertainty of $0.1 \mathrm{~m} \mathrm{~s}^{-1}$ on the cup anemometers amounts to $5 \%$ at $2 \mathrm{~m} \mathrm{~s}^{-1}$ (see Supplementary Material for more information). This means that the measurement uncertainties will be high for these data and they are thus excluded. Data above $10 \mathrm{~m} \mathrm{~s}^{-1}$ are likewise excluded to reduce calculation time. This is not expected to influence the results, since these data constitute a very small fraction of the total data. 


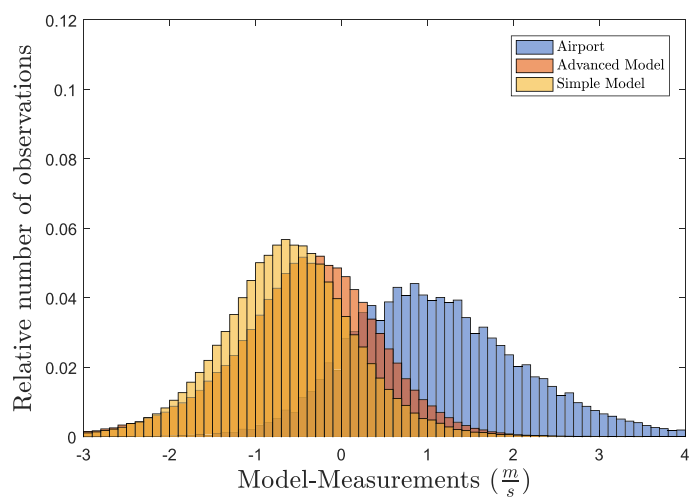

(a) Kastrup $\rightarrow$ HCOE (17 years)

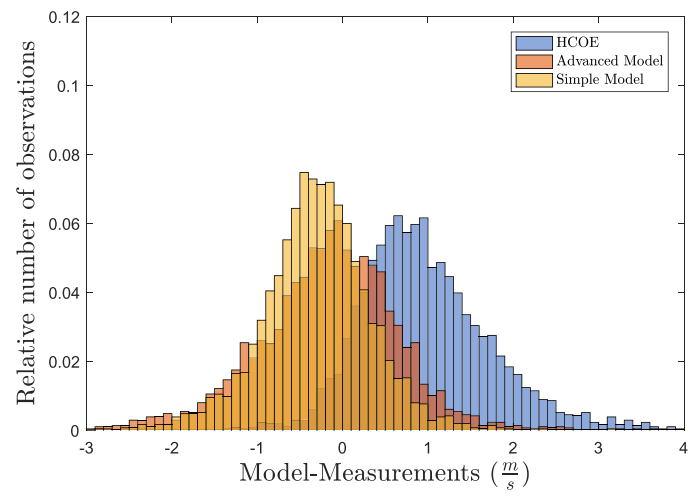

(c) $\mathrm{HCOE} \rightarrow \mathrm{HCAB}(5$ months)

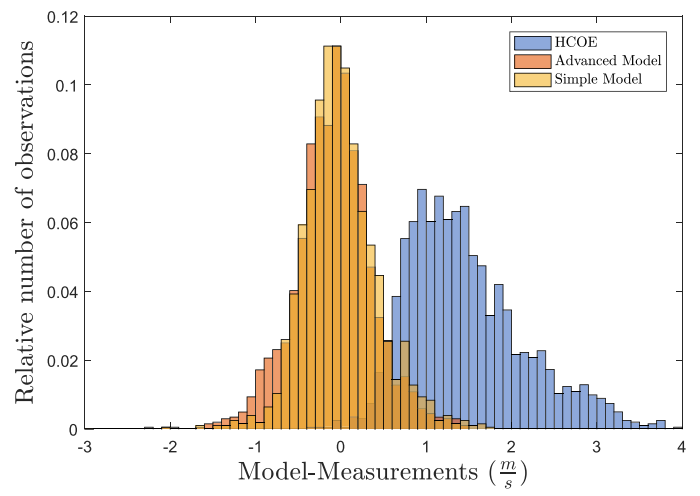

(e) $\mathrm{HCOE} \rightarrow$ Jagtvej (5 months)

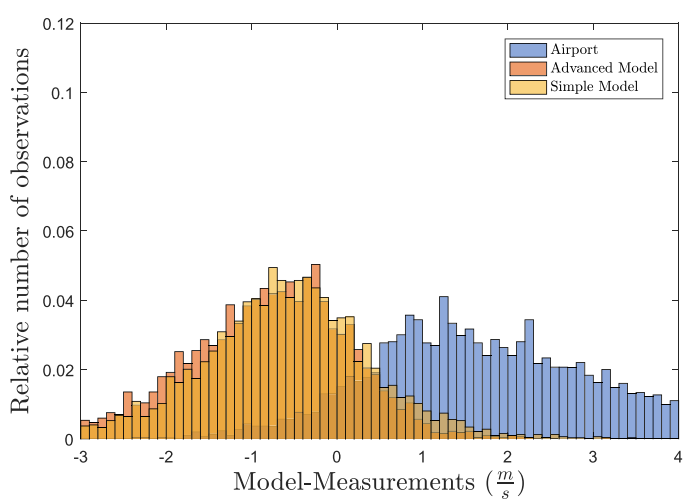

(b) Kastrup $\rightarrow \mathrm{HCAB}$ (5 months)

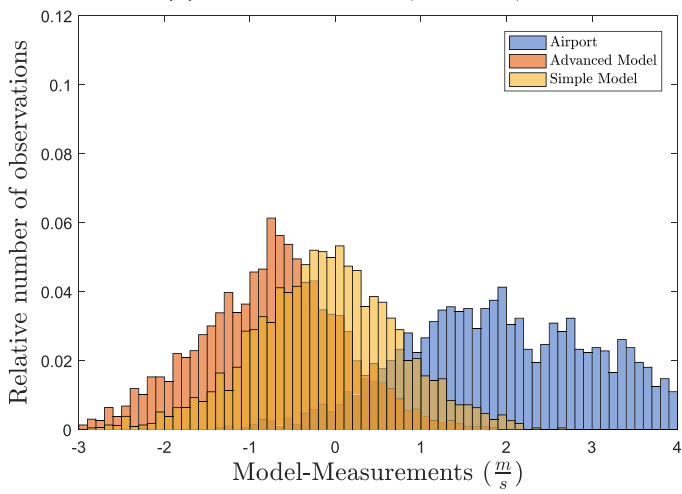

(d) Kastrup $\rightarrow$ Jagtvej (5 months)

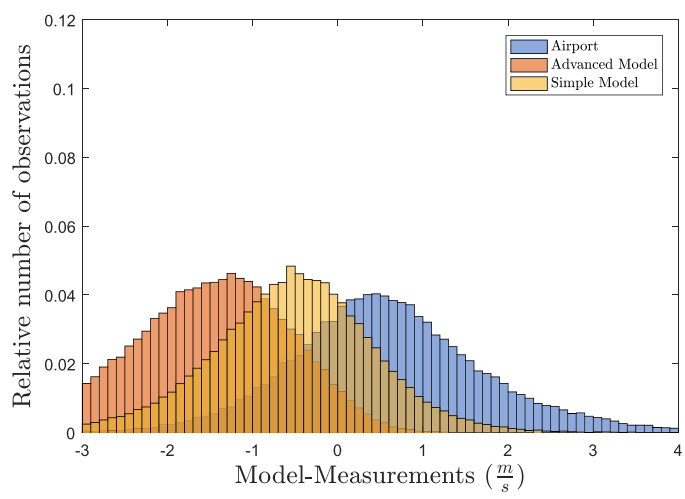

(f) Tirstrup $\rightarrow$ Aarhus (10 years)

Figure 6. Histograms of respectively the input minus the measurements or the models minus the measurements.

Measurements. Long term wind speed measurements have been performed in Copenhagen, Denmark at the H. C. Oersted Institute, Copenhagen University (HCOE) and in Aarhus at the town hall under the Danish Air Quality Monitoring Programme for the period 1994 to 2010 for HCOE and for the period 2001 to 2010 for Aarhus $^{38}$. The details of the meteorological instruments used in the present study can be found in Table 1 in the Supplementary Material. The cup anemometers are located on $7 \mathrm{~m}$ masts on urban rooftops. This is referred to as the mast type measurements in Table 1 in the Supplementary Material. The uncertainty in the wind speed measurements is described in the Supplementary Material. The wind direction is measured using two types of wind vanes (Risoe P2633A or Vector Instruments W200P) with a wind direction resolution of $0.2^{\circ}$ and a specified uncertainty of $\pm 3^{\circ}$ (in steady winds over $5 \mathrm{~m} \mathrm{~s}^{-1}$ ). The data scanning frequency of both the wind speed and the wind direction measurements is $0.1 \mathrm{~Hz}$. These data is subsequently averaged to hourly wind speeds and directions. The data is subsequently quality controlled using the procedure described in ${ }^{16}$.

For the present study, a wind measurement campaign was designed. The campaign consisted of setting up two sonic anemometers on rooftops in Copenhagen, Denmark at respectively Jagtvej and H. C. Andersens Boulevard $(\mathrm{HCAB})$ to measure the wind speed close to the roof level. The sonic anemometers were mounted $4 \mathrm{~m}$ to $5 \mathrm{~m}$ above roof level. This is referred to as the roof type measurements in Table 1. This height was chosen in line with the aim of the study being to measure the wind speed in the roughness sublayer. The measurements were 
performed over a five months period from December 20, 2014 to May 1, 2015. The two locations were chosen, since extensive air pollution studies at the two streets have been performed in the past (e.g. ${ }^{4-46}$ ). The sonic anemometer at Jagtvej was a RM Young 81000 with a specified accuracy of $\pm 1 \%$ or $\pm 0.05 \mathrm{~m} \mathrm{~s}^{-1}$ below $30 \mathrm{~m} \mathrm{~s}^{-1}$ and $\pm 3 \%$ above $30 \mathrm{~m} \mathrm{~s}^{-1}$, and the sonic anemometer at HCAB was a Metek USA-1 with a specified accuracy of $0.1 \mathrm{~m} \mathrm{~s}^{-1}$ or $2 \%$ at $5 \mathrm{~m} \mathrm{~s}^{-1}$. Both sonics were sampling at $10 \mathrm{~Hz}$. The measurements were despiked by removing measurements with accelerations larger than $5 \sigma$ (where $\sigma$ is the standard deviation of the hourly average) or velocities larger than $10 \sigma$. Following the recommendation from ${ }^{47}$, no rotations were applied to the sonics. This is because, the flow in the urban roughness sublayer is highly three-dimensional. The assumptions for the rotation techniques are therefore not fulfilled. The measurements were subsequently averaged to hourly values with the wind speed being the sum of the $u, v$, and $w$ components.

The wind speed measurements from Kastrup Airport and Tirstrup Airport were obtained from National Climatic Data Center, National Oceanic and Atmospheric Administration (ncdc.noaa.gov). This means that the measurements were not performed by the authors but represent standard meteorological data publicly available. The wind speeds were obtained as METeorological Aerodrome Report (METAR) measurements. These have a wind direction resolution of $10^{\circ}$ and a wind speed resolution of $0.45 \mathrm{~m} \mathrm{~s}^{-1}(1 \mathrm{mile} / \mathrm{h})$. These were subsequently averaged to hourly wind speeds and directions. The details of the measurements are summed up in Table 1, and the location of the measurement stations can be seen in the attached Google Maps file.

Wind speed model. The wind speed model should simulate the hourly mean wind speed at the height and location of interest, in line with the approaches used for the regulatory air pollution models. This is a different approach compared with all the studies in Table 4 in the Supplementary Material, in that these are modelling various forms of area-averaged winds. Because of the three-dimensional nature of the urban roughness sublayer, both the measurements and the modelled wind speed cannot be claimed to be representative of a wider area. The input to the model should come from a measured wind speed either at a non-urban location, e.g. an airport, or an urban mast. As a general principle, all parametrizations are applied in the model with the published parameters in order not to add increased parameter uncertainty to the model.

Extrapolation scheme. The wind speed is extrapolated from one location to another using the principle by ${ }^{31}$ as follows:

1. The "macrowind" $\left(S_{h}\right)$, also called the "effective geostrophic wind", is calculated from a measured wind speed through an upwards vertical extrapolation. It should be emphasised that the macrowind is not necessarily equal to the synoptic geostrophic wind speed derived from macroscale pressure gradients ${ }^{31}$.

2. The speed and direction of the macrowind are assumed to be constant over the area of extrapolation thus allowing horizontal extrapolation.

3. The urban wind speed is calculated from the macrowind through a downwards vertical extrapolation.

Model summary. The model can be summarised in the following steps:

1. The Geographical preprocessor generates a map of $z_{0}$ and $d$ as a function of wind direction. The input is maps of land use and building geometry in raster format. This procedure is described in the Supplementary Material.

2. The upwind distances from the station to the onset of the IBLs are calculated from the map of $z_{0}$, as described in the Supplementary Material.

3. The heights of the individual IBLs $(\delta)$, at the location of the station, are calculated using the approach of ${ }^{48}$.

4. The blending height is calculated based on the distance to the onset of the closest IBL to the station.

5. The upwind footprint of the mixed layer is calculated as described in the Supplementary Material.

6. The boundary layer height $\left(h_{u b l}\right)$ is calculated using the approach of ${ }^{49}$.

7. $S_{h}$ is calculated with repeated using the approach of ${ }^{31}$ (upwards extrapolation).

8. $S_{h}$ is assumed to be constant between the two locations thus providing horizontal extrapolation.

9. The process is repeated for the downwards extrapolation.

10. The results are subsequently averaged over a $30^{\circ}$ wind direction interval to account for hourly wind direction meandering.

Details of the calculations in the individual step can be found in the Supplementary Material. For the downwards extrapolation, Items 5 to 7 are iterated since $h_{u b l}$ is a function of the wind speed. The above described wind speed extrapolation model was implemented in Matlab by the authors. Differences between the present modelling approach and that of previous studies are discussed in the Supplementary Material.

Model without influence of the upwind terrain. To analyse the influence of the upwind terrain, a simpler model was developed based on the same principles as described above. The simple model also uses the extrapolation scheme from ${ }^{31}$ but applies several simplifying assumptions:

- $\quad z_{0}$ and $d$ are calculated from the building height of the building where the anemometer is located $\left(h_{r}\right)$ rather than using the geographical preprocessor. The equations for $z_{0}$ and $d$ are simplified expressions of ${ }^{50}$ as follows:

$$
z_{0}=0.15 h_{r}
$$




$$
d=0.7 h_{r}
$$

It is recommended that the maximum allowed building height for the parametrisation is $20 \mathrm{~m}^{50}$. This recommendation has been implemented in the model. The airport stations are assigned a constant roughness length of $0.005 \mathrm{~m}$ (short grass) following ${ }^{51}$. The vertical extrapolation is performed with one roughness length and displacement height instead of using the above described IBL approach. This means that upwind influence on the station is not taken into account.

- The horizontal extrapolation height is assumed to be $4 h_{r}$ following the recommendation by ${ }^{52}$ thus also removing the wind speed dependency of the model.

This approach is referred to as the simple model in the results section.

\section{References}

1. Albrecht, F. Untersuchungen der vertikalen luftzirkulation in der grossstadt. Meteorologische Zeitung 50, 93-98 (1933).

2. Oke, T. R. Review of Urban Climatology 1968-1973. Technical Note 134, World Meteorological Organization (1974).

3. Oke, T. R. Review of Urban Climatology 1973-1976. Technical Note 169, World Meteorological Organization (1979).

4. Rotach, M. W. J. Turbulence Within and Above and Urban Canopy. Ph.D. thesis, ETH Zürich (1991).

5. Vignati, E., Berkowicz, R. \& Hertel, O. Comparison of air quality in streets of copenhagen and milan, in view of the climatological conditions. Science of The Total Environment 189, 467-473, https://doi.org/10.1016/0048-9697(96)05247-3 (1996).

6. Fisher, B. et al. Meteorology applied to urban air pollution problems - Final Report COST Action 715 (2005).

7. Luhar, A. K., Venkatram, A. \& Lee, S.-M. On relationships between urban and rural near-surface meteorology for diffusion applications. Atmospheric Environment 40, 6541-6553 (2006).

8. Wyszogrodzki, A. A., Miao, S. \& Chen, F. Evaluation of the coupling between mesoscale-wrf and les-eulag models for simulating fine-scale urban dispersion. Atmospheric Research 118, 324-345, https://doi.org/10.1016/j.atmosres.2012.07.023 (2012).

9. Mortarini, L. et al. Characterization of wind meandering in low-wind-speed conditions. Boundary-Layer Meteorology 161, 165-182, https://doi.org/10.1007/s10546-016-0165-6(2016).

10. You, T., Wu, R., Huang, G. \& Fan, G. Regional meteorological patterns for heavy pollution events in beijing. Journal of Meteorological Research 31, 597-611, https://doi.org/10.1007/s13351-017-6143-1 (2017).

11. Droste, A. M., Steeneveld, G. J. \& Holtslag, A. A. M. Introducing the urban wind island effect. Environmental Research Letters 13, 094007, https://doi.org/10.1088/1748-9326/aad8ef (2018).

12. Sunderland, K. M., Mills, G. \& Conlon, M. F. Estimating the wind resource in an urban area: A case study of micro-wind generation potential in Dublin, Ireland. Journal of Wind Engineering and Industrial Aerodynamics 118, 44-53 (2013).

13. Millward-Hopkins, J., Tomlin, A., Ma, L., Ingham, D. \& Pourkashanian, M. Mapping the wind resource over UK cities. Renewable Energy 55, 202-211, https://doi.org/10.1016/j.renene.2012.12.039 (2013).

14. Triantafyllou, A. et al. Evaluation of an atmospheric model with surface and abl meteorological data for energy applications in structured areas. Theoretical and Applied Climatology 135, 1227-1242, https://doi.org/10.1007/s00704-018-2429-1, Cited By 0 (2019).

15. Drew, D. R., Barlow, J. F. \& Lane, S. E. Observations of wind speed profiles over greater london, uk, using a doppler lidar. Journal of Wind Engineering and Industrial Aerodynamics 121, 98-105, https://doi.org/10.1016/j.jweia.2013.07.019 (2013).

16. Ottosen, T.-B. et al. A Parameter Estimation and Identifiability Analysis Methodology Applied to a Street Canyon Air Pollution Model. Environmental Modelling \& Software 84, 165-176 (2016).

17. Venkatram, A., Isakov, V., Pankratz, D. \& Yuan, J. Relating plume spread to meteorology in urban areas. Atmospheric Environment 39, 371-380, https://doi.org/10.1016/j.atmosenv.2004.08.045 (2005).

18. Hertel, O. \& Berkowicz, R. Modelling Pollution from Traffic in a Street Canyon. Evaluation of Data and Model Development. Tech. Rep. 129, National Environmental Research Institute, DMU LUFT-A (1989).

19. Berkowicz, R. Modelling street canyon pollution: model requirements and expectations. International Journal of Environment and Pollution 8, 609-619 (1997).

20. Ottosen, T.-B. Improved Local Air Quality Modelling for Complex Urban Landscapes. Ph.D. thesis, Intstitute for Chemical Engineering, Biotechnology, and Environmental Technology, University of Southern Denmark (2016).

21. Barlow, J. F. Progress in observing and modelling the urban boundary layer. Urban Climate 10, Part 2, 216-240, https://doi. org/10.1016/j.uclim.2014.03.011, ICUC8: The 8th International Conference on Urban Climate and the 10th Symposium on the Urban Environment (2014).

22. Tartakovsky, D., Stern, E. \& Broday, D. M. Evaluation of modeled wind field for dispersion modeling. Atmospheric Research 166, 150-156, https://doi.org/10.1016/j.atmosres.2015.07.004 (2015).

23. Krähenmann, S., Walter, A., Brienen, S., Imbery, F. \& Matzarakis, A. High-resolution grids of hourly meteorological variables for germany. Theoretical and Applied Climatology 131, 899-926, https://doi.org/10.1007/s00704-016-2003-7 (2018).

24. Barlow, J. F., Harrison, J., Robins, A. G. \& Wood, C. R. A wind-tunnel study of flow distortion at a meteorological sensor on top of the BT tower, london, UK. Journal of Wind Engineering and Industrial Aerodynamics 99, 899-907, https://doi.org/10.1016/j. jweia.2011.05.001, 9th \{UK\} Conference on Wind Engineering (September, 2010) (2011).

25. Liu, C.-H. \& Wong, C. C. On the pollutant removal, dispersion, and entrainment over two-dimensional idealized street canyons. Atmospheric Res. 135-136, 128-142, https://doi.org/10.1016/j.atmosres.2013.08.006 (2014).

26. Ricci, A. et al. Large-scale forcing effects on wind flows in the urban canopy: Impact of inflow conditions. Sustainable Cities and Society 42, 593-610, https://doi.org/10.1016/j.scs.2018.08.012 (2018).

27. de Wit, M., Stathopoulos, T. \& Wisse, J. Airport wind speeds used for the design in urban environments: the eindhoven case. Journal of Wind Engineering and Industrial Aerodynamics 90, 1289-1298, https://doi.org/10.1016/S0167-6105(02)00258-1, 3rd EuropeanAfrican Conference on Wind Engineering (Part two) (2002).

28. Drew, D., Barlow, J. \& Cockerill, T. Estimating the potential yield of small wind turbines in urban areas: A case study for greater london, UK. Journal of Wind Engineering and Industrial Aerodynamics 115, 104-111, https://doi.org/10.1016/j.jweia.2013.01.007 (2013).

29. Weekes, S. \& Tomlin, A. Evaluation of a semi-empirical model for predicting the wind energy resource relevant to small-scale wind turbines. Renewable Energy 50, 280-288, https://doi.org/10.1016/j.renene.2012.06.053 (2013).

30. Kent, C. W., Grimmond, C., Gatey, D. \& Barlow, J. F. Assessing methods to extrapolate the vertical wind-speed profile from surface observations in a city centre during strong winds. Journal of Wind Engineering and Industrial Aerodynamics 173, 100-111, https:// doi.org/10.1016/j.jweia.2017.09.007 (2018).

31. Wieringa, J. Roughness-dependent geographical interpolation of surface wind speed averages. Quarterly Journal of the Royal Meteorological Society 112,867-889, https://doi.org/10.1002/qj.49711247316 (1986).

32. Mertens, S. The energy yield of roof mounted wind turbines. Wind Engineering 27, 507-518, https://doi. org/10.1260/030952403773617472, http://wie.sagepub.com/content/27/6/507.full.pdf+html (2003).

33. Heath, M. A., Walshe, J. D. \& Watson, S. J. Estimating the potential yield of small building-mounted wind turbines. Wind Energy 10 , 271-287, https://doi.org/10.1002/we.222 (2007). 
34. Deaves, D. Computations of wind flow over changes in surface roughness. Journal of Wind Engineering and Industrial Aerodynamics 7, 65-94, https://doi.org/10.1016/0167-6105(81)90068-4 (1981).

35. Soriano, C. et al. Comparison of urban and rural wind speeds. In Rotach, M. W., Fisher, B. \& Piringer, M. (eds) Extended abstracts of the Workshop on Urban Boundary Layer Parameterisations, 41-50 (2001).

36. Best, M. et al. Small-scale Wind Energy - Technical Report. Tech. Rep., Met Office (2008).

37. Barlow, J. F., Rooney, G. G., von Hünerbein, S. \& Bradley, S. G. Relating urban surface-layer structure to upwind terrain for the salford experiment (salfex). Boundary-Layer Meteorology 127, 173-191, https://doi.org/10.1007/s10546-007-9261-y (2008).

38. Ellermann, T. et al. The Danish Air Quality Monitoring Programme. Annual Summary for 2012. Tech. Rep. Scientific Report from DCE. no. 67, Arhus University, DCE - Danish Centre for Environment and Energy (2013).

39. Seinfeld, J. H. \& Pandis, S. N. Atmospheric Chemistry and Physics (John Wiley \& Sons, Inc., 2006).

40. Fortuniak, K., Kłysik, K. \& Wibig, J. Urban-rural contrasts of meteorological parameters in łódź. Theoretical and Applied Climatology 84, 91-101, https://doi.org/10.1007/s00704-005-0147-y (2006).

41. Wood, C. et al. Turbulent flow at $190 \mathrm{~m}$ height above london during 2006-2008: A climatology and the applicability of similarity theory. Boundary-Layer Meteorology 137, 77-96, https://doi.org/10.1007/s10546-010-9516-x (2010).

42. Gryning, S.-E., Batchvarova, E., Brümmer, B., Jørgensen, H. \& Larsen, S. On the extension of the wind profile over homogeneous terrain beyond the surface boundary layer. Boundary-Layer Meteorology 124, 251-268, https://doi.org/10.1007/s10546-007-9166-9 (2007).

43. Pelliccioni, A., Monti, P., Gariazzo, C. \& Leuzzi, G. Some characteristics of the urban boundary layer above rome, italy, and applicability of monin-obukhov similarity. Environmental Fluid Mechanics 12, 405-428, https://doi.org/10.1007/s10652-012-9246-3 (2012).

44. Berkowicz, R., Palmgren, F., Hertel, O. \& Vignati, E. Using measurements of air pollution in streets for evaluiation of urban air quality - meteorological analysis and model calculations. The Science of the Total Environment 189/190, 259-265 (1996).

45. Ketzel, M. et al. Evaluation of the Street Pollution Model OSPM for Measurements at 12 Streets Stations Using a Newly Developed and Freely Available Evaluation Tool. Journal of Civil \& Environmental Engineering S:1 (2012).

46. Berkowicz, R., Winther, M. \& Ketzel, M. Traffic pollution modelling and emission data. Environmental Modelling \& Software 21, 454-460 (2006).

47. Christen, A. Atmospheric Turbulence and Surface Energy Exchange in Urban Environments. Ph.d.-thesis, PhilosophischNaturwissenschaftlichen Fakultät der Universität Basel (2005).

48. Wood, D. H. Internal Boundary Layer Growth Following a Step Change in Surface Roughness. Boundary Layer Meteorology 22, 241-244 (1981).

49. Rossby, C.-G. \& Montgomery, R. B. The Layer of Frictional Influence in Wind and Ocean Currents. Papers in Physical Oceanography and Meteorology 3, 1-101 (1935).

50. Hanna, S. R. \& Britter, R. E. Wind Flow and Vapor Cloud Dispersion at Industrial and Urban Sites (American Institute of Chemical Engineers, 2002)

51. Oke, T. R. Boundary Layer Climates, 2 edn. (Routledge, 1987).

52. Oke, T. R. Initial Guidance to Obtain Representative Meteorological Observations at Urban Sites. Tech. Rep. Report nr. 81, World Meteorological Organization (2006).

\section{Acknowledgements}

The authors wish to express their gratitude to Claus Nordstrøm for information about the measurement procedures employed in the Danish National Air Quality Monitoring Programme, to Per Løfstrøm for the development of the program to process the raw sonic data, and to Ebba Dellwik for guidance on the analysis of local turbulence measurements. The HPC resources and services used in this work were provided by the IT Research Computing group in Texas A\&M University at Qatar. IT Research Computing is funded by the Qatar Foundation for Education, Science and Community Development (http://www.qf.org.qa). This publication was made possible by a NPRP award [NPRP 7-674-2-252] from the Qatar National Research Fund (a member of The Qatar Foundation). The statements made herein are solely the responsibility of the authors. The Danish EPA under the Danish Ministry of Environment provided funding for the long term meteorological measurements. The project was supported by the DCE - Danish Centre for Environment and Energy, Aarhus University Grant number: 2015-20440.

\section{Author Contributions}

All authors participated in setting up the study concept and the study design. T.-B.O., M.K. and K.E.K. designed the measurement campaign, and the measurements were performed by T.-B.O. and M.K. T.-B.O., M.K. and K.E.K. analysed the data to assess the validity of the assumptions. T.-B.O. developed and validated the wind speed models with input from all the authors. H.S. and K.E.K. obtained funding for the study. T.-B.O. wrote the article manuscript. All the co-authors participated in the interpretation of the results, provided critical comments to the manuscript, and read and approved the final manuscript.

\section{Additional Information}

Supplementary information accompanies this paper at https://doi.org/10.1038/s41598-019-50033-2.

Competing Interests: The authors declare no competing interests.

Publisher's note Springer Nature remains neutral with regard to jurisdictional claims in published maps and institutional affiliations.

Open Access This article is licensed under a Creative Commons Attribution 4.0 International License, which permits use, sharing, adaptation, distribution and reproduction in any medium or format, as long as you give appropriate credit to the original author(s) and the source, provide a link to the Creative Commons license, and indicate if changes were made. The images or other third party material in this article are included in the article's Creative Commons license, unless indicated otherwise in a credit line to the material. If material is not included in the article's Creative Commons license and your intended use is not permitted by statutory regulation or exceeds the permitted use, you will need to obtain permission directly from the copyright holder. To view a copy of this license, visit http://creativecommons.org/licenses/by/4.0/.

(C) The Author(s) 2019 